\title{
PROLONGATION ALGEBRA AND BÄCKLUND TRANSFORMATIONS OF DRINFELD-SOKOLOV SYSTEM OF EQUATIONS
}

\author{
Ayşe Karasu(Kalkanli) \\ and \\ Ísmet Yurduşen \\ Department of Physics \\ Middle East Technical University \\ 06531 Ankara, Turkey \\ e-mail:akarasu@metu.edu.tr
}

\begin{abstract}
We show that the Drinfeld-Sokolov system of equations has a non-trivial prolongation structure. The closure process for prolongation algebra gives rise to the $s l(4, c)$ algebra which is used to derive the scattering problem for the system of equations under consideration. The non-trivial new Bäcklund transformations and some explicit solutions are given.
\end{abstract}




\section{Introduction}

The systems of nonlinear partial differential equations are encountered in fundamental particle physics, plasma and fluid dynamics, statistical mechanics, many areas of solid state physics, protein dynamics, laser and fiber optics[1]. These equations are usually solved by the use of approximation techniques, but the range of applicability and usefulness of these solutions increase the interest on the exact solutions and methods of testing for complete integrability. During the past three decades the developments in mathematical physics show that the completely integrable systems of nonlinear partial differential equations have very rich mathematical structures such as the existence of Lax pair, bi-Hamiltonian structures, recursion operators and applicability of inverse scattering methods. One of the effective methods to test integrability is the prolongation structure technique of Wahlquist and Estabrook[2]. This geometrical method immediately proceeds with an attempt to construct the linear spectral problem or another device, such as Bäcklund transformations. If we are successful we have not only tested our system of equations for complete integrability but also constructed a device with which to integrate the system. Dodd and Fordy [3],[4] made the method more algorithmic and put it in an algebraic, instead of differential geometric framework. The first step is to start with a differential equation and derive a set of generators and relations for an incomplete Lie algebra. The second step is to complete this Lie algebra and find a finite matrix representation for the derived set of generators. Then, one expects that Bäcklund transformations, which are relations among solutions of the nonlinear differential equations under consideration, may be obtained. For a review the reader is referred to an article by Harrison[5] and references quoted therein.

The integrable systems appear not one at a time, but in big families which are called hierarchies. First, Korteweg-de Vries(KdV)-hierarchy was invented and then infinitely many generalized KdV-hierarchies were found. They were unified to a single one large Kadomtsev- Petviashvili(KP)-hierarchy[6]. 
Very recently, it is shown by Gürses and Karasu [7] that the system of equations

$$
\begin{aligned}
& u_{t}=-u_{x x x}+6 u u_{x}+6 v_{x} \\
& v_{t}=2 v_{x x x}-6 u v_{x}
\end{aligned}
$$

admits a recursion operator and a bi-Hamiltonian structure,therefore it has constants of motion. The system (1.1) belongs to a type of equations which are called "quasi-polynomial flows". The Lax pair for this system was first given by Drinfeld and Sokolov [8] and later by Bogoyavlenskii [9]. Under the scale transformations, this system of equations reduces to a special case of the KP-hierarchy which was given by Satsuma and Hirota [10]. They also gave the one-soliton solutions. Recently, auto-Backlund transformations and certain analytical solutions are obtained by Tian and Gao [11] via Painléve analysis. In this work, we used the prolongation method to derive the linear scattering problem for the system (1.1). We obtained Bäcklund transformations by using pseudopotentials.

\section{Prolongation Structure}

By introducing the variables $p=u_{x}, q=v_{x}, r=p_{x}, s=q_{x}$, the system of equations (1.1) can be represented by the set of 2 -forms,

$$
\begin{aligned}
& \alpha_{1}=d u \wedge d t-p d x \wedge d t \\
& \alpha_{2}=d p \wedge d t-r d x \wedge d t \\
& \alpha_{3}=d v \wedge d t-q d x \wedge d t \\
& \alpha_{4}=d q \wedge d t-s d x \wedge d t \\
& \alpha_{5}=d u \wedge d x-d r \wedge d t+6(u p+q) d x \wedge d t \\
& \alpha_{6}=d v \wedge d x+2 d s \wedge d t-6 u q d x \wedge d t
\end{aligned}
$$

which constitutes a closed ideal $I$, such that $d I \subset I$. 
We extend the ideal $I$ by adding to it the system of 1 -forms,

$$
w^{A}=d y^{A}+F^{A} d x+G^{A} d t, \quad A=1, \ldots, N
$$

where $F^{A}$ and $G^{A}$ are functions of $\left(u, v, p, q, r, s, y^{A}\right)$, which are assumed in the form $F^{A}=F_{B}^{A} y^{B}, G^{A}=G_{B}^{A} y^{B}$. The extended ideal must be closed under exterior differentiations. This requirement gives the set of partial differential equations for $F^{A}$ and $G^{A}$. Dropping the indices for simplicity, we have

$$
\begin{gathered}
F_{p}=F_{q}=F_{r}=F_{s}=0, \quad G_{s}=2 F_{v}, \quad G_{r}=-F_{u}, \\
p G_{u}+q G_{v}+r G_{p}+s G_{q}-6(u p+q) F_{u}+6 u q F_{v}-[F, G]=0
\end{gathered}
$$

where

$$
[F, G]=F^{B} \frac{\partial G}{\partial y^{B}}-G^{B} \frac{\partial F}{\partial y^{B}}
$$

Next, we integrate equations (2.3). In reaching the results we equate the coefficients of quadratic terms in F to zero, since these coefficients are in the centre of prolongation algebra[4]. The final result is,

$$
\begin{aligned}
F= & X_{1}+X_{2} u+X_{3} v \\
G= & X_{0}+\left(-r+3 u^{2}+6 v\right) X_{2}+2(s-3 u v) X_{3}-p X_{4}-u X_{5} \\
& -\frac{u^{2}}{2} X_{6}+2 q X_{7}+2 v X_{8}+v^{2} X_{9}+2 u v X_{10}
\end{aligned}
$$

where $X_{0}, X_{1}, X_{2}, X_{3}$ are constants of integration, depending on $y^{A}$ only. The remaining elements are,

$$
\begin{array}{lll}
X_{4}=\left[X_{1}, X_{2}\right], & X_{5}=\left[X_{1}, X_{4}\right], & X_{6}=\left[X_{2}, X_{4}\right], \\
X_{7}=\left[X_{1}, X_{3}\right], & X_{8}=\left[X_{1}, X_{7}\right], & X_{9}=\left[X_{3}, X_{7}\right], \\
& & X_{10}=\left[X_{2}, X_{7}\right] .
\end{array}
$$


The integrability conditions impose following restrictions on $X_{i}(i=1, \ldots, 10)$,

$$
\begin{aligned}
{\left[X_{2}, X_{3}\right]=0, \quad\left[X_{2}, X_{6}\right]=0, \quad\left[X_{3}, X_{9}\right]=0, \quad\left[X_{1}, X_{0}\right] } & =0 \\
{\left.\left[X_{3}, X_{4}\right]=6 X_{3}-2 X_{10}\right]+2\left[X_{3}, X_{10}\right] } & =0 \\
{\left[X_{2}, X_{10}\right]-\frac{1}{4}\left[X_{3}, X_{6}\right] } & =0 \\
{\left[X_{1}, X_{6}\right]+2\left[X_{2}, X_{5}\right]-6 X_{4} } & =0 \\
{\left[X_{1}, X_{9}\right]+2\left[X_{3}, X_{8}\right] } & =0 \\
{\left[X_{1}, X_{5}\right]-\left[X_{2}, X_{0}\right] } & =0 \\
2\left[X_{1}, X_{8}\right]+\left[X_{3}, X_{0}\right]+6 X_{4} & =0 \\
{\left[X_{1}, X_{10}\right]+\left[X_{2}, X_{8}\right]-\frac{1}{2}\left[X_{3}, X_{5}\right]-3 X_{7} } & =0 .
\end{aligned}
$$

Using the Jacobi identities we obtain further relations:

$$
\begin{array}{r}
{\left[X_{1}, X_{9}\right]=\left[X_{3}, X_{8}\right]=0, \quad\left[X_{2}, X_{9}\right]=\left[X_{3}, X_{10}\right]=0} \\
{\left[X_{2}, X_{10}\right]=\left[X_{3}, X_{6}\right]=0, \quad\left[X_{2}, X_{7}\right]=\left[X_{3}, X_{4}\right]} \\
{\left[X_{1}, X_{10}\right]-\left[X_{4}, X_{7}\right]-\left[X_{2}, X_{8}\right]=0,} \\
{\left[X_{1}, X_{6}\right]=\left[X_{2}, X_{5}\right]=2 X_{4}, \quad\left[X_{4}, X_{6}\right]=-2 X_{6},} \\
{\left[X_{3}, X_{5}\right]+\left[X_{7}, X_{4}\right]+2\left[X_{1}, X_{10}\right]=6 X_{7} .}
\end{array}
$$

In order to find the Lie algebra generated by $\mathrm{F}$ and matrix representations of the generators $\left\{X_{i}\right\}_{0}^{10}$, we follow the strategy of Dodd-Fordy[4]. It can be summarized as follows:

1. Locate elements of the center of the algebra. Assuming the algebra to be (semi-)simple equate these elements with zero.

2. Locate a nilpotent and semi-simple element.

3. Embed these elements in a simple Lie algebra $g$.

4. Express the remaining generators of the prolongation algebra as linear combinations of a suitable basis of $g$.

5. Use the fundamental representation of $g$ to generate a linear scattering problem. 
First we reduce the number of elements. By using equations (2.6), (2.7) and (2.8) we get

$$
X_{9}=0, \quad X_{6}=2 X_{2}, \quad X_{10}=2 X_{3} .
$$

Next, we locate nilpotent and neutral elements. Equations (2.6) and (2.9) give that $X_{2}$ is nilpotent and $X_{4}$ is neutral element. Let us note that the system of equations in (1.1) has the following scale symmetry

$$
x \rightarrow \lambda^{-1} x, \quad t \rightarrow \lambda^{-3} t, \quad u \rightarrow \lambda^{2} u, \quad v \rightarrow \lambda^{4} v
$$

which implies that the elements $X_{i}$ must satisfy

$$
\begin{aligned}
& X_{0} \rightarrow \lambda^{3} X_{0}, \quad X_{1} \rightarrow \lambda X_{1}, \quad X_{2} \rightarrow \lambda^{-1} X_{2}, \quad X_{3} \rightarrow \lambda^{-3} X_{3}, \\
& X_{4} \rightarrow X_{4}, \quad X_{5} \rightarrow \lambda X_{5}, \quad X_{7} \rightarrow \lambda^{-2} X_{7}, \quad X_{8} \rightarrow \lambda^{-1} X_{8} .
\end{aligned}
$$

By using the basis elements, we try to embed the prolongation algebra into $s l(n+1, c)$. Starting from the cases $n=1,2$, we found that $s l(2, c)$ and $s l(3, c)$ can not be the whole algebra. The simplest non-trivial closure is in terms of $\operatorname{sl}(4, c)$. Without giving the details here we present the results:

$$
\begin{gathered}
X_{1}=\left(\begin{array}{cccc}
0 & 1 & 0 & 0 \\
0 & 0 & 2 & 0 \\
0 & 0 & 0 & 1 \\
\frac{\lambda^{4}}{2} & 0 & 0 & 0
\end{array}\right), \\
X_{3}=\left(\begin{array}{cccc}
0 & 0 & 0 & 0 \\
0 & 0 & 0 & 0 \\
0 & 0 & 0 & 0 \\
-\frac{1}{2} & 0 & 0 & 0
\end{array}\right), \\
X_{5}=\left(\begin{array}{cccc}
0 & -2 & 0 & 0 \\
0 & 0 & 4 & 0 \\
0 & 0 & 0 & -2 \\
\lambda^{4} & 0 & 0 & 0
\end{array}\right),
\end{gathered}
$$




$$
X_{8}=\left(\begin{array}{cccc}
0 & 0 & 0 & 0 \\
-1 & 0 & 0 & 0 \\
0 & 1 & 0 & 0 \\
0 & 0 & -1 & 0
\end{array}\right), \quad X_{0}=\left(\begin{array}{cccc}
0 & 0 & 0 & -8 \\
-4 \lambda^{4} & 0 & 0 & 0 \\
0 & -2 \lambda^{4} & 0 & 0 \\
0 & 0 & -4 \lambda^{4} & 0
\end{array}\right)
$$

These generators satisfy the following commutation relations:

$$
\begin{aligned}
& {\left[X_{2}, X_{4}\right]=2 X_{2}, \quad\left[X_{2}, X_{3}\right]=0, \quad\left[X_{3}, X_{4}\right]=2 X_{3} \text {, }} \\
& {\left[X_{1}, X_{2}\right]=X_{4}, \quad\left[X_{1}, X_{3}\right]=X_{7}, \quad\left[X_{4}, X_{7}\right]=0,} \\
& {\left[X_{3}, X_{7}\right]=0, \quad\left[X_{1}, X_{7}\right]=X_{8}, \quad\left[X_{3}, X_{8}\right]=0,} \\
& {\left[X_{2}, X_{7}\right]=2 X_{3}, \quad\left[X_{1}, X_{4}\right]=X_{5}, \quad\left[X_{2}, X_{5}\right]=2 X_{4} \text {, }} \\
& {\left[X_{3}, X_{5}\right]=2 X_{7}, \quad\left[X_{2}, X_{8}\right]=2 X_{7}, \quad\left[X_{0}, X_{1}\right]=0,} \\
& {\left[X_{2}, X_{0}\right]=\left[X_{1}, X_{5}\right], \quad\left[X_{3}, X_{0}\right]=-6 X_{4}-2\left[X_{1}, X_{8}\right]}
\end{aligned}
$$

By substituting the matrix representations of the generators into equation (2.5) we form the matrices $\mathrm{F}$ and $\mathrm{G}$. Using equations $y_{x}=-F y, y_{t}=-G y$ ,the linear scattering problem can be obtained as,

$$
\begin{aligned}
& \left(\begin{array}{l}
y^{1} \\
y^{2} \\
y^{3} \\
y^{4}
\end{array}\right)_{x}=\left(\begin{array}{cccc}
0 & -u & 0 & -\frac{\lambda^{4}}{2}+\frac{v}{2} \\
-1 & 0 & 0 & 0 \\
0 & -2 & 0 & -u \\
0 & 0 & -1 & 0
\end{array}\right)\left(\begin{array}{l}
y^{1} \\
y^{2} \\
y^{3} \\
y^{4}
\end{array}\right) \\
& \left(\begin{array}{l}
y^{1} \\
y^{2} \\
y^{3} \\
y^{4}
\end{array}\right)_{t}=\left(\begin{array}{cccc}
p & 4 \lambda^{4}-4 v+r-2 u^{2} & q & \lambda^{4} u+s-u v \\
-2 u & -p & 2 \lambda^{4}-2 v & -q \\
0 & 4 u & p & 4 \lambda^{4}-4 v+r-2 u^{2} \\
8 & 0 & -2 u & -p
\end{array}\right)\left(\begin{array}{c}
y^{1} \\
y^{2} \\
y^{3} \\
y^{4}
\end{array}\right)
\end{aligned}
$$

which is equivalent to the scalar Lax equation

$$
L \psi=\left(\partial^{4}-2 u \partial^{2}-2 u_{x} \partial-u_{x x}+u^{2}+v\right) \psi=\lambda^{4} \psi
$$


where $\psi=y^{4}$ and $\lambda=$ constant. The corresponding time evolution of $\psi$ is

$$
\psi_{t}=\left(-4 \partial^{3}+6 u \partial+3 u_{x}\right) \psi
$$

\section{Bäcklund Transformations}

Within the prolongation scheme, Bäcklund transformations can be derived by assuming the new solution variables as functions of old ones and the ratios of pseudopotentials[5]. For this purpose, let us define new variables

$$
\alpha=\frac{y^{1}}{y^{4}}, \quad \beta=\frac{y^{2}}{y^{4}}, \quad \gamma=\frac{y^{3}}{y^{4}} .
$$

By using equations (2.14) and (2.15) we can find the equations satisfied by $\alpha, \beta$ and $\gamma$,

$$
\begin{aligned}
\alpha_{x} & =\alpha \gamma-u \beta+\frac{1}{2}\left(v-\lambda^{4}\right) \\
\beta_{x} & =-\alpha+\beta \gamma \\
\gamma_{x} & =\gamma^{2}-2 \beta-u \\
\alpha_{t} & =-8 \alpha^{2}+2(p+2 u \gamma) \alpha+\left(4 \lambda^{4}-4 v+r-2 u^{2}\right) \beta+q \gamma+\left(\lambda^{4} u+s-u v\right) \\
\beta_{t} & =-2 u \alpha+2(u \gamma-4 \alpha) \beta+2\left(\lambda^{4}-v\right) \gamma-q \\
\gamma_{t} & =2 u \gamma^{2}+2(p-4 \alpha) \gamma+4 u \beta+\left(4 \lambda^{4}-4 v+r-2 u^{2}\right)
\end{aligned}
$$

The compatibility conditions $\alpha_{x t}=\alpha_{t x}$ and $\gamma_{x t}=\gamma_{t x}$ hold if $u$ and $v$ satisfy equation (1.1), while $\beta_{x t}=\beta_{t x}$ holds automatically. One can easily check that the function $\beta$ satisfies the following equation,

$$
\beta_{t}-2 \beta_{x x x}+6\left(u+2 \gamma_{x}\right) \beta_{x}=0
$$

This means that

$$
\begin{aligned}
& \tilde{u}=u+2 \gamma_{x}, \\
& \tilde{v}=c_{1} \beta+c_{2}
\end{aligned}
$$


are the new solutions of equations (1.1) if

$$
\left(4 \beta^{2}+c_{1} \beta\right)_{x}=0
$$

where $c_{1}$ and $c_{2}$ are constants. We note that the same results for $\tilde{u}$ and $\tilde{v}$ were obtained when we followed the $\operatorname{step}(3)$ of ref.[5]. By seting $y_{4}=\psi$ and using equations $(2.14),(2.15)$ and (3.1) we obtain

$$
\begin{aligned}
& \tilde{u}=u-2 \frac{\psi_{x x}}{\psi}+2 \frac{\psi_{x}^{2}}{\psi^{2}} \\
& \tilde{v}=c_{1}\left(-\frac{u}{2}+\frac{\psi_{x x}}{\psi}\right)+c_{2} .
\end{aligned}
$$

Here, $u$ is a known solution of equations (1.1) and $\psi$ is the solution of equations $(2.16),(2.17)$ satisfying the condition

$$
\left\{\frac{1}{2 \psi^{2}}\left[\psi_{x x}-u \psi\right]\left[2\left(\psi_{x x}-u \psi\right)+c_{1} \psi\right]\right\}_{x}=0 .
$$

which is equivalent to (3.5).

Next, we consider the simple case $u=v=0$ as the known solution of (1.1) and find a new solution. With this choice, we find that

$$
\psi=d_{1} e^{-\lambda\left(4 \lambda^{2} t-x\right)}+d_{2} e^{\lambda\left(4 \lambda^{2} t-x\right)}+d_{3} e^{i \lambda\left(4 \lambda^{2} t+x\right)}+d_{4} e^{-i \lambda\left(4 \lambda^{2} t+x\right)}
$$

is a solution of equations (2.16) and (2.17) where $d_{1}, d_{2}, d_{3}$ and $d_{4}$ are constants. Substituting (3.8) into condition (3.7) we obtain two sets of solutions for $\psi$ whereas $\left\{d_{1}=d_{2}=0, c_{1}=4 \lambda^{2}\right\}$ and $\left\{d_{3}=d_{4}=0, c_{1}=-4 \lambda^{2}\right\}$.

The respective solutions for $\tilde{u}$ are

$$
\begin{aligned}
& \tilde{u}_{1}=\frac{8 d_{3} d_{4} \lambda^{2} e^{2 i \lambda\left(4 \lambda^{2} t+x\right)}}{\left[d_{4}+d_{3} e^{2 i \lambda\left(4 \lambda^{2} t+x\right)}\right]^{2}} \\
& \tilde{u}_{2}=\frac{-8 d_{1} d_{2} \lambda^{2} e^{2 \lambda\left(4 \lambda^{2} t-x\right)}}{\left[d_{1}+d_{2} e^{2 \lambda\left(4 \lambda^{2} t-x\right)}\right]^{2}}
\end{aligned}
$$


where in both cases $\tilde{v}=c_{2}-2 \lambda^{4}=$ constant. Thus, starting from trivial background, we obtained the one soliton solution of $\mathrm{KdV}$ equation with $\tilde{v}=$ constant .

In order to find the more general Bäcklund transformations for system (1.1), we assume that new solutions $\tilde{U}$ and $\tilde{V}$ are functions of old variables $u, v, p, q, r, s$ and $\alpha, \beta, \gamma$ which are the ratios of prolongation variables satisfying the equations (3.2). After some straightforward but long calculations we obtained the following results:

$$
\begin{aligned}
\tilde{U} & =\frac{\Omega}{2}-\Delta-\frac{2}{\gamma}(\alpha+2 \beta \gamma), \\
\tilde{V} & =\Theta_{x x}-\frac{\Delta}{\gamma} \Theta_{x}-\Theta^{2},
\end{aligned}
$$

where

$$
\begin{aligned}
\Omega & =-\frac{1}{\gamma^{2}}\left(p \gamma+u^{2}+4 \beta u+4 \beta^{2}-4 \alpha \gamma\right) \\
\Delta & =2 \beta-2 \gamma^{2}+u \\
\Theta & =\frac{1}{4 \gamma^{2}}\left[-u^{2}+2\left(\gamma^{2}-2 \beta\right) u-4\left(\alpha \gamma+\beta^{2}\right)\right]
\end{aligned}
$$

and $\Theta$ must satisfy the condition

$$
\Theta_{t}=2 \Theta_{x x x}+3 \Omega \Theta_{x}
$$

Thus, if any solutions $u$ and $v$ to Drinfeld-Sokolov system of equations are known and if $\alpha, \beta, \gamma$ are solutions of (3.2) satisfying the condition (3.12), then $\tilde{U}$ and $\tilde{V}$ are the new solutions of (1.1).

If we take the trivial seed solutions $u=v=0$, we get

$$
\begin{aligned}
& \tilde{U}=-\frac{2 \beta^{2}}{\gamma^{2}}+2\left(\gamma^{2}-2 \beta\right), \\
& \tilde{V}=-\frac{1}{\gamma^{4}}\left(17 \beta^{4}+8 \beta^{3} \gamma^{2}-10 \beta^{2} \alpha \gamma+\alpha^{2} \gamma^{2}\right)+\left(\frac{\lambda^{4}}{2}+\frac{c_{0}}{18}\right),
\end{aligned}
$$


with the condition

$$
\beta\left[-56 \beta^{4}+48 \beta^{2} \gamma \alpha-3 \beta \gamma^{2} \lambda^{4}-8 \gamma^{2} \alpha^{2}+\gamma^{4} \lambda^{4}\right]=0
$$

By setting $y^{4}=\psi$, one can write these expressions in terms of the solutions of Lax equations $(2.16),(2.17)$ and see that our results are more general than the given by Tian and Gao[11].

As an example, we consider the simple case, $u=v=0$ as the known solutions of (1.1) and we obtain the following two sets of explicit solutions:

$$
\tilde{U}=\frac{2 c_{1}^{2}}{\left(c_{1} x+c_{2}\right)^{2}}, \quad \tilde{V}=\frac{c_{0}}{18} .
$$

and

$$
\begin{aligned}
\tilde{U} & =\frac{\left.30\left(c_{1}+x^{2}\right)\left\{4 x\left[5 c_{1}\left[2\left(24 t-x^{3}\right)-3 c_{1} x\right]-\left(c_{2}+3 x^{5}\right)\right]+15\left(x^{2}+c_{1}\right)^{3}\right]\right\}}{\left\{5 c_{1}\left[2\left(24 t-x^{3}\right)-3 c_{1} x\right]-\left(c_{2}+3 x^{5}\right)\right\}^{2}} \\
\tilde{V} & =-\frac{120 x\left[c_{2}+3 x^{5}+15 c_{1}^{2} x-10 c_{1}\left(24 t-x^{3}\right]^{3}\right.}{\left\{5 c_{1}\left[2\left(24 t-x^{3}\right)-3 c_{1} x\right]-\left(c_{2}+3 x^{5}\right)\right\}^{4}}
\end{aligned}
$$

where $c_{0}, c_{1}, c_{2}$ are constants.

As we observed that there are no solitary wave solutions belonging to the class (3.13). Very recently, all the special solutions of (1.1) are obtained by Karasu and Sakovich [12].

\section{Conclusion}

In this work we have rederived the linear scattering problem for DrinfeldSokolov system of equations by using the prolongation algorithm. We found the auto-Bäcklund transformations and some exact solutions of these equations. The system can be integrated by the method of inverse scattering problem associated with the fourth order Lax operator L, which was developed by Iwasaki [13]. It is known that the most general Bäcklund transformation would be the one which utilizes the infinite dimensional algebra 
and not all of finite algebras give rise to Bäcklund transformations. Without searching if the incomplete algebra given in (2.7) is finite or infinite dimensional we used a finite dimensional representation of prolongation algebra and derived non-trivial Bäcklund transformations. Thus, the methods given in [4] and [5] are quite useful from the practical point of view, for the systems of nonlinear partial differential equations. Finally, we note that a close connection between some stationary flows associated with fourth-order Lax operators and generalisations of some integrable Hamiltonian systems with quartic potentials is known [14]. The equations (2.16) and (2.17) can be considered in this context.

We would like to thank Dr.Atalay Karasu and Dr.Sergei Sakovich for useful discussions. We also thank to anonymous referee for valuable

comments. This work is supported in part by the Scientific and Technical Research Council of Turkey (TUBITAK).

\section{References}

[1] Fordy,A.P.(ed.),Soliton Theory: A Survey of Results, Manchester University Press, (1990).

[2] Wahlquist, H.D. and Estabrook, F.B., J.Math.Phys.,16,1(1975).

[3] Dodd,R.,Fordy,A.,Physics Letters, A 89, 168(1982).

[4] Dodd,R.,Fordy,A.,Proc.R.Soc.Lond.,A 385, 389(1983).

[5] Harrison,K.,Journal of Nonlinear Mathematical Physics,2, 201(1995). 
[6] Date,E., Jimbo,M., Kashiwara,M., Miwa,T., In Non-linear Integrable Systems, Classical and Quantum Theory. Proceedings of RIMS Symposium, Singapore,39(1983).

[7] Gürses,M., Karasu,A., Physics Letters, A 251,347(1999).

[8] Drinfeld,V.G., Sokolov,V.V., Proceedings of S.L. Sobolev Seminar, Novosibirsk, 2, 5(1981) (in Russian).

[9] Bogoyavlenskii,O.I., Russian Math. Surveys ,45, 1(1990).

[10] Satsuma,J.,Hirota,R., J.Phys.Soc.J., 51, 3390(1982).

[11] Tian,B., Gao,Y.,Physics Letters, A 208, 193(1995).

[12] Karasu(Kalkanlı),A., Sakovich,S.Yu., Preprint arXiv:nlin.SI/0102001(2001).

[13] Iwasaki, K., Japan J. Math.14,1(1988).

[14] Baker,S., Enolskii,V.Z., Fordy,A.P.,Physics Letters,A 201, 167(1995). 